\title{
MATHEMATICAL STUDY OF A NONLINEAR NEURON MULTI-DENDRITIC MODEL
}

\author{
BY \\ PIERLUIGI COLLI \\ University of Pavia, Italy
}

\begin{abstract}
A mathematical model of potential spreading along neuron dendrites is proposed to describe the synaptic transmissions in the so-called cerebellar granule cells, which consist of a nearly spherical soma emitting a finite number of dendrites. The model accounts for the nonlinear dependence of the NMDA receptors, located at the virtual ends of any dendrite, upon the voltage. The corresponding initialboundary value problem is formulated in the framework of Sobolev spaces. Existence and uniqueness of a weak solution are proved along with regularity results ensuring that the solution is classical.
\end{abstract}

1. Introduction. In the physiological study of synaptic transmissions between neurons, two interesting facts were experimentally observed. The first one regards the existence of some central neurons, named cerebellar granule cells, consisting of a nearly spherical soma which emits four dendrites on the average (see, e.g., $[9,10]$ ). Each dendrite receives at its virtual end one excitatory synapse, which is therefore located far away from the somatic recording site. Secondly, the synapses express one class of receptors, the so-called NMDA receptors, whose dependence on voltage is nonlinear (cf., e.g., $[8,7,1,4])$ and which can then interact with membrane potential in the living neurons.

Then it becomes important to simulate and predict the interactions between voltage changes originating from one or more synaptic inputs and the synaptic receptors located on the other dendrites. To this concern we refer to [3], where a mathematical model of potential spreading along dendrites, assuming linear boundary conditions, is proposed and identified with respect to experimental situations. In this paper we are interested in analysing and solving the related mathematical problem in the general case of nonlinear relationships between potentials and currents at the synaptic receptors (as stated in, e.g., $[1,4]$ ).

Usually two experimental situations are distinguished, according to whether the membrane potential or the membrane current is fixed in the soma (allowing the related

Received May 15, 1992.

1991 Mathematics Subject Classification. Primary 35K60, 92C20; Secondary 35K50, 35A15.

This work has been supported by M.U.R.S.T. (fondi per la ricerca scientifica) and by the Istituto di Analisi Numerica of C.N.R., Pavia, Italy. 
current or potential to change). These conditions are known as voltage-clamp and current-clamp, respectively, and can be experimentally obtained by the patch-clamp whole-cell recording technique applied to cerebellar slices (see [4]). Here we study the mathematical problem related to the latter situation, i.e., current-clamp, while the results concerning the former can easily be recovered by our analysis.

Referring to $[3,2,11]$ for more details and precise dimensions of data and variables, let us just introduce the basic equations and conditions of the model. Let $N \geq 1$ be the number of dendrites connected to the same soma, and let $l_{i}$ be the length of the dendrite denoted by the index $i$, for $i=1, \ldots, N$. Following [11], we regard any dendrite as a one-dimensional continuum reduced to an interval $\left[0, l_{i}\right]$, where the (common) point $x=0$ yields the soma position and $x=l_{i}$ represents the (free) synaptic end. Taking an arbitrary time $T>0$ and letting $\left.u_{i}:\right] 0, l_{i}[\times] 0, T[\rightarrow \mathbf{R}, i=1, \ldots, N$, be the dendritic potentials, the voltage transmission in the dendrite $i$ is described by the following well-known cable equation (see, e.g., $[2,11,3])$ :

$$
\left.\tau_{i} \partial_{t} u_{i}-\lambda_{i}^{2} \partial_{x x} u_{i}+u_{i}=0 \text { in }\right] 0, l_{i}[\times] 0, T[,
$$

where $\partial_{t}, \partial_{x x}$ denote the partial derivative with respect to the variable $\left.t \in\right] 0, T[$ and the second derivative with respect to $x \in] 0, l_{i}\left[\right.$, respectively. Besides $\tau_{i}, \lambda_{i}$, $i=1, \ldots, N$, represent positive constants depending on the physical properties of the neuronal membrane and (possibly) assuming different values according to the corresponding dendrite. Then Eq. (1.1) yields a system of $N$ linear parabolic equations which will be coupled with suitable initial and boundary conditions. As initial conditions we take

$$
\left.u_{i}(x, 0)=u_{i 0}(x), \quad x \in\right] 0, l_{i}[, \quad i=1, \ldots, N,
$$

where the rest potentials $u_{i 0}, i=1, \ldots, N$, are experimentally determined. Next, as all dendrites are attached to the soma, the following $N-1$ boundary conditions have to be satisfied:

$$
\left.u_{1}(0, t)=u_{2}(0, t)=\cdots=u_{n}(0, t), \quad t \in\right] 0, T[.
$$

By considering the current-clamp condition, the current intensity in the soma is prescribed (cf. [3]), i.e.,

$$
\left.-\sum_{i=1}^{N} a_{i} \partial_{x} u_{i}(0, t)=\gamma(t), \quad t \in\right] 0, T[.
$$

Here $a_{i}, i=1, \ldots, N$, are known positive constants related to the conductance of the dendrites and the operator $-\partial_{x}$ stands for the outward normal derivatives on the first ends (always corresponding to $x=0$ ) of the boundaries. Then it remains to state conditions on the other ends that have to account for the NMDA receptors activation during synaptic inputs. According to $[8,7,1,4]$, we assume nonlinear relationships between the current and the applied voltage

$$
\left.\partial_{x} u_{i}\left(l_{i}, t\right)+g_{i}\left(u_{i}\left(l_{i}, t\right)\right)=0, \quad t \in\right] 0, T[, \quad i=1, \ldots, N,
$$


where $g_{i}: \mathbf{R} \rightarrow \mathbf{R}, i=1, \ldots, N$, are given continuous functions, experimentally obtained in the voltage-clamp situation. Referring to [4, Fig. 3, p. 469] for various graphs proposed for these functions, let us describe some properties of $g_{i}$. The behaviour in a neighbourhood of $-\infty$ may be of two different types: either $-\infty \swarrow g_{i}(\xi)$ or $0 \backslash g_{i}(\xi)$ as $\xi \searrow-\infty$. In the former case $g_{i}$ increases until a relative maximum, then it exhibits a local minimum and goes to $+\infty$ as $\xi \nearrow+\infty$. Otherwise, $g_{i}$ has an absolute minimum, where it takes a negative value, and $g_{i}(\xi) \nearrow+\infty$ as $\xi \nearrow+\infty$. Both cases are considered in our analysis, where we just assume that (if $g_{i}$ is differentiable) the derivative $g_{i}^{\prime}$ is bounded from below by a real number (actually our assumption (H4) is weaker, see Remark 2.1).

As a first step toward the validation of the model, in this paper we prove an existence and uniqueness result for the problem (1.1)-(1.5) in the framework of Hilbert spaces. In order to show the existence of a solution, we introduce a time discretization of the problem and find a unique discrete solution by solving suitable nonlinear equations with the help of monotonicity arguments. Then we state some a priori estimates and pass to the limit by applying compactness methods to deal with the nonlinearities. Uniqueness is deduced by contradiction. Moreover, the regularity of the solution is discussed. For a numerical analysis, which applies our time discretization procedure and uses a spectral method in space, we refer to [5]. Numerical tests reproducing experimental conditions and comparison results are reported in [3].

As already mentioned, the problem (1.1)-(1.5) reproduces the current-clamp experimental condition. If one wants to describe the voltage-clamp situation, the only differences consist in removing the boundary condition (1.4) and prescribing the potential value in the soma, that is,

$$
\left.u_{1}(0, t)=\cdots=u_{n}(0, t)=\mu(t), \quad t \in\right] 0, T[.
$$

Then it is a standard matter to see that the related problem (1.1)-(1.2) and (1.5)-(1.6) consists of $N$ independent problems which are easily solvable once the mathematical study of (1.1)-(1.5) has been performed. Thus here we will not consider the voltageclamp problem: actually, the same techniques can be used and the analysis turns out to be simpler.

The outline of the paper is the following. A variational formulation of the problem (1.1)-(1.5) is presented in the next section along with our main result. In Sec. 3 we show that there exists at most one solution. Section 4 is devoted to the approximation of the problem: a backward finite differences scheme is used. In Sec. 5 we deduce the a priori estimates, and in Sec. 6 we pass to the limit by the arguments outlined above. Finally, Sec. 7 contains some remarks about regularity of the solution and possible extensions of our results.

2. Variational formulation and main result. In this section we shall introduce a weak formulation of the problem (1.1)-(1.5) and state our existence and uniqueness theorem. To this aim, we consider the following function spaces:

$$
\begin{aligned}
\mathbf{H} & :=L^{2}\left(0, l_{1}\right) \times \cdots \times L^{2}\left(0, l_{N}\right), \\
\mathbf{V} & :=\left\{\mathbf{v}=\left(v_{1}, \ldots, v_{N}\right): v_{i} \in H^{1}\left(0, l_{i}\right), v_{i}(0)=v_{j}(0) \quad \text { for } i, j=1, \ldots, N\right\} .
\end{aligned}
$$


Owing to Eqs. (1.1) and conditions (1.3), we will require that any weak solution $\mathbf{u}=\left(u_{1}, \ldots, u_{N}\right)$ of $(1.1)-(1.5)$ satisfies $\mathbf{u}(\cdot, t) \in \mathbf{V}$ for a.e. $\left.t \in\right] 0, T[$. Henceforth we shall use the notation

$$
\mathscr{G}_{0}(\mathbf{v}):=v_{1}(0)=\cdots=v_{n}(0) \quad \forall \mathbf{v}=\left(v_{1}, \ldots, v_{N}\right) \in \mathbf{V} .
$$

Let us formally deduce the variational equality for our problem. Take then an arbitrary $\mathbf{v}=\left(v_{1}, \ldots, v_{N}\right) \in \mathbf{V}$, multiply Eq. (1.1) by $v_{i}$, and integrate it over ]0, $l_{i}[$. By a simple integration by parts and by Eq. (1.5) we obtain

$$
\begin{gathered}
\tau_{i} \int_{0}^{l_{i}} \partial_{t} u_{i} v_{i}+\lambda_{i}^{2} \int_{0}^{l_{i}} \partial_{x} u_{i} \partial_{x} v_{i}+\lambda_{i}^{2} g_{i}\left(u_{i}\left(l_{i}, \cdot\right)\right) v_{i}\left(l_{i}\right) \\
+\lambda_{i}^{2} \partial_{x} u_{i}(0, t) v_{i}(0)+\int_{0}^{l_{i}} u_{i} v_{i}=0
\end{gathered}
$$

for $i=1, \ldots, N$, a.e. in $] 0, T\left[\right.$. Multiplying this equation by $a_{i} / \lambda_{i}^{2}$ and summing up with respect to $i$, owing to (1.4) and (2.2) we have that

$$
\begin{aligned}
& \sum_{i=1}^{N} \frac{a_{i} \tau_{i}}{\lambda_{i}^{2}} \int_{0}^{l_{i}} \partial_{t} u_{i} v_{i}+\sum_{i=1}^{N} a_{i} \int_{0}^{l_{i}} \partial_{x} u_{i} \partial_{x} v_{i}+\sum_{i=1}^{N} \frac{a_{i}}{\lambda_{i}^{2}} \int_{0}^{l_{i}} u_{i} v_{i} \\
& \quad+\sum_{i=1}^{N} a_{i} g_{i}\left(u_{i}\left(l_{i}, \cdot\right)\right) v_{i}\left(l_{i}\right)=\gamma \mathscr{T}_{0}(\mathbf{v})
\end{aligned}
$$

a.e. in $] 0, T\left[\right.$. Therefore, we shall look for $\mathbf{u}=\left(u_{1}, \ldots, u_{N}\right)$ satisfying Eq. (2.4) for any $\mathbf{v} \in \mathbf{V}$ and fulfilling the initial conditions (2.2).

In order to make our statement more precise, we introduce the following bilinear and continuous forms on $\mathbf{H} \times \mathbf{H}$ :

$$
\langle\mathbf{v}, \mathbf{w}\rangle:=\sum_{i=1}^{N} \frac{a_{i} \tau_{i}}{\lambda_{i}^{2}} \int_{0}^{l_{i}} v_{i} w_{i}
$$

and on $\mathbf{V} \times \mathbf{V}$ :

$$
(\mathbf{v}, \mathbf{w}):=\sum_{i=1}^{N} a_{i}\left(\int_{0}^{l_{i}} \partial_{x} v_{i} \partial_{x} w_{i}+\frac{1}{\lambda_{i}^{2}} \int_{0}^{l_{i}} u_{i} v_{i}\right) .
$$

It is a standard matter to check that $\mathbf{H}$ and $\mathbf{V}$ are Hilbert spaces endowed with the scalar products defined above and the corresponding norms. Also, by identifying $\mathbf{H}$ with its dual space, it follows easily (cf., e.g., [6] for analogous procedures) that $\mathbf{V} \subset \mathbf{H} \subset \mathbf{V}^{\prime}$ with dense and continuous injections. Next, let us just remark that

$$
\mathbf{V} \subset C^{0}\left(\left[0, l_{1}\right]\right) \times \cdots \times C^{0}\left(\left[0, l_{N}\right]\right),
$$

so that (see also the definition (2.3)) for a.e. $t \in[0, T]$ the term $\gamma(t) \mathscr{T}_{0}(\mathbf{v})$ of Eq. (2.4) yields a linear and continuous operator on $\mathbf{V}$.

Before stating the variational formulation of the problem, we list our assumptions on the data. Let

(H1) $\mathbf{u}_{0}=\left(u_{01}, \ldots, u_{0 N}\right) \in \mathbf{V}$,

(H2) $\gamma \in W^{1,1}(0, T)$, 
(H3) $g_{i} \in C^{0}(\mathbf{R})$,

(H4) $\exists d_{i} \geq 0$ : for any $\xi, \eta \in \mathbf{R}$ satisfying $\xi>\eta$ one has

$$
g_{i}(\xi)-g_{i}(\eta) \geq-d_{i}(\xi-\eta),
$$

hold for $i=1, \ldots, N$. Then it is easy to see that, owing to (H3) and (2.7), in the equality (2.4) also the term $\sum_{i=1}^{N} a_{i} g_{i}\left(u_{i}\left(l_{i}, \cdot\right)\right) v_{i}\left(l_{i}\right)$ makes sense.

REMARK 2.1. Note that (H4) is a classical one-sided Lipschitz condition. It is equivalent to requiring that the sum of $g_{i}$ with a large multiple of the identity be monotone. In addition, if the function $g_{i}$ is differentiable in some point $\xi \in \mathbf{R}$, then (H4) entails $g_{i}^{\prime}(\xi) \geq-d_{i}$. On the other hand, let us point out that a wide class of functions, including the nonlinear relationships between current and voltage fitting the experimental data (see, e.g., $[1,4])$, satisfy the assumptions (H3)-(H4): regarding monotonicity and behaviour at infinity, several different cases can be considered.

Finally, taking into account (2.5)-(2.6), a precise variational formulation of the problem (1.1) $-(1.5)$ reads as follows.

Problem (P). Find $\mathbf{u}=\left(u_{1}, \ldots, u_{N}\right) \in H^{1}(0, T ; \mathbf{H}) \cap L^{2}(0, T ; \mathbf{V})$ satisfying

$$
\begin{gathered}
\mathbf{u}(0)=\mathbf{u}_{0}, \\
\left.\left\langle\partial_{t} \mathbf{u}, \mathbf{v}\right\rangle+(\mathbf{u}, \mathbf{v})+\sum_{i=1}^{N} a_{i} g_{i}\left(u_{i}\left(l_{i}, \cdot\right)\right) v_{i}\left(l_{i}\right)=\gamma \mathscr{T}_{0}(\mathbf{v}) \quad \forall \mathbf{v} \in \mathbf{v}, \text { a.e. in }\right] 0, T[.
\end{gathered}
$$

Here is our main result.

THEOREM 2.1. Under the assumptions $(\mathrm{H} 1)-(\mathrm{H} 4)$ there exists one and only one solution u of Problem (P). Moreover,

$$
\mathbf{u} \in C^{0}([0, T] ; \mathbf{V}) \cap L^{2}\left(0, T ; H^{2}\left(0, l_{1}\right) \times \cdots \times H^{2}\left(0, l_{N}\right)\right) .
$$

RemarK 2.2. Thanks to (2.10), from Eq. (2.9) it follows that the solution $\left(u_{1}, \ldots\right.$, $u_{N}$ ) of Problem (P) satisfies Eqs. (1.1) a.e. in $] 0, l_{i}[\times] 0, T[$, for $i=1, \ldots, N$. Indeed, in order to recover Eq. (1.1) for $i=1$, it suffices to take $\mathbf{v}=\left(v_{1}, 0, \ldots, 0\right)$, with an arbitrary $v_{1} \in H_{0}^{1}\left(0, l_{1}\right)$, as a test function in Eq. (2.9), and then integrate by parts in space. Also, since $\mathbf{u} \in C^{0}([0, T] ; \mathbf{V})$, by $(2.7),(2.8)$, and $(2.2)$ we have that Eqs. (1.2) and (1.3) hold for any $x \in\left[0, l_{i}\right]$ and any $t \in[0, T]$, respectively. Moreover, by choosing suitable test functions in Eq. (2.9) and using Eqs. (1.1), it is not difficult to show that the boundary conditions (1.4)-(1.5) are satisfied for a.e. $t \in] 0, T$. Finally, let us note that, owing to (2.10), (H2), (H3), and (2.7), by comparison in Eq. (2.9) we obtain $\partial_{t} \mathbf{u} \in C^{0}\left([0, T] ; \mathbf{V}^{\prime}\right)$.

3. Uniqueness. Here we will prove that Problem (P) has at most one solution. First, let us prepare a simple result.

Lemma 3.1. There exists a positive constant $C$ such that for any $\mathbf{v}=\left(v_{1}, \ldots, v_{N}\right) \in$ $\mathbf{V}$ and for $i=1, \ldots, N$ one has

$$
\left|v_{i}(x)\right|^{2} \leq C\left(1+\varepsilon^{-1}\right) \int_{0}^{l_{i}}\left|v_{i}\right|^{2}+\varepsilon \int_{0}^{l_{i}}\left|\partial_{x} v_{i}\right|^{2} \quad \forall x \in\left[0, l_{i}\right], \forall \varepsilon>0 .
$$


Proof. Since $v_{i} \in H^{1}\left(0, l_{i}\right)$ (cf. (2.2)), the inequality

$$
\left|v_{i}(x)\right|^{2} \leq\left|v_{i}(y)\right|^{2}+2\left|\int_{x}^{y} v_{i} \partial_{x} v_{i}\right|
$$

holds for any $x, y \in\left[0, l_{i}\right]$. By integrating it over $\left[0, l_{i}\right]$ with respect to $y$, we deduce that

$$
l_{i}\left|v_{i}(x)\right|^{2} \leq \int_{0}^{l_{i}}\left|v_{i}\right|^{2}+2 l_{i} \int_{0}^{l_{i}}\left|v_{i}\right|\left|\partial_{x} v_{i}\right|
$$

Then (3.1) follows from a well-known estimate applied to the second term of the right-hand side. We can take, for instance, $C=\max \left\{1,1 / l_{1}, \ldots, 1 / l_{N}\right\}$.

Now assume by contradiction that there exist two different solutions $\tilde{\mathbf{u}}$ and $\hat{\mathbf{u}}$ of Problem (P) and set $\mathbf{u}=\tilde{\mathbf{u}}-\hat{\mathbf{u}}$. We take the difference of Eqs. (2.9) corresponding to $\tilde{\mathbf{u}}$ and $\hat{\mathbf{u}}$, respectively, and then choose $\mathbf{v}=\mathbf{u}$ and integrate in time accounting for the initial conditions (2.8). Thus we obtain

$$
\frac{1}{2}\|\mathbf{u}(t)\|_{\mathbf{H}}^{2}+\int_{0}^{t}\|\mathbf{u}(s)\|_{\mathbf{V}}^{2} d s=-\int_{0}^{t} \sum_{i=1}^{N} a_{i}\left(g_{i}\left(\tilde{u}_{i}\left(l_{i}, s\right)\right)-g_{i}\left(\hat{u}_{i}\left(l_{i}, s\right)\right)\right) u_{i}\left(l_{i}, s\right) d s
$$

for any $t \in[0, T]$. In order to estimate the right-hand side of Eq. (3.2), we start to remark that the assumption (H4) can be equivalently formulated as

$$
\left(g_{i}(\xi)-g_{i}(\eta)\right)(\xi-\eta) \geq-d_{i}|\xi-\eta|^{2} \quad \forall \xi, \eta \in \mathbf{R} .
$$

Then, from (3.2)-(3.3) it follows that

$$
\frac{1}{2}\|\mathbf{u}(t)\|_{\mathbf{H}}^{2}+\int_{0}^{t}\|\mathbf{u}(s)\|_{\mathbf{V}}^{2} d s \leq \int_{0}^{t} \sum_{i=1}^{N} a_{i} d_{i}\left|u_{i}\left(l_{i}, s\right)\right|^{2} d s
$$

and, thanks to Lemma 3.1 and to the definitions $(2.5)-(2.6)$, there is a constant $\bar{C}$ such that

$$
\int_{0}^{t} \sum_{i=1}^{N} a_{i} d_{i}\left|u_{i}\left(l_{i}, s\right)\right|^{2} d s \leq \bar{C} \int_{0}^{t}\|\mathbf{u}(s)\|_{\mathbf{H}}^{2} d s+\frac{1}{2} \int_{0}^{t}\|\mathbf{u}(s)\|_{\mathbf{V}}^{2} d s,
$$

where, for instance, $\bar{C}=\max \left\{d_{i} \lambda_{i}^{2} C\left(1+2 d_{i}\right) / \tau_{i}, i=1, \ldots, N\right\}$. Therefore, we have

$$
\|\mathbf{u}(t)\|_{\mathbf{H}}^{2}+\int_{0}^{t}\|\mathbf{u}(s)\|_{\mathbf{V}}^{2} d s \leq 2 \bar{C} \int_{0}^{t}\|\mathbf{u}(s)\|_{\mathbf{H}}^{2} d s \quad \forall t \in[0, T] .
$$

Hence, by applying the Gronwall lemma, we conclude that $\mathbf{u}=\tilde{\mathbf{u}}-\hat{\mathbf{u}}=\mathbf{0}$, that is a contradiction.

Remark 3.1. Concerning the assumptions on the data of Problem (P), let us point out that $(\mathrm{H} 4)$ is the only hypothesis we have considered in proving uniqueness.

4. Approximation. In this section we approximate Problem (P) by using an implicit time discretization procedure. Therefore, let $M$ be an arbitrary positive integer and let $h:=T / M$ denote the time step in our backward finite differences scheme. Setting for $j=0,1, \ldots, M$

$$
\gamma^{j}:=\gamma(j h)
$$


we consider the following

Problem $\left(\mathbf{P}_{h}\right)$. Find $\mathbf{U}^{j}=\left(U_{1}^{j}, \ldots, U_{N}^{j}\right) \in \mathbf{V}, j=0,1, \ldots, M$, satisfying

$$
\mathbf{U}^{0}=\mathbf{u}_{0}
$$

and, for $j=1, \ldots, M$,

$$
\left\langle\frac{\mathbf{U}^{j}-\mathbf{U}^{j-1}}{h}, \mathbf{v}\right\rangle+\left(\mathbf{U}^{j}, \mathbf{v}\right)+\sum_{i=1}^{N} a_{i} g_{i}\left(U_{i}^{j}\left(l_{i}\right)\right) v_{i}\left(l_{i}\right)=\gamma^{j} \mathscr{T}_{0}(\mathbf{v}) \quad \forall \mathbf{v} \in \mathbf{V} .
$$

We shall prove that, for a time step $h$ sufficiently small, Problem $\left(\mathrm{P}_{h}\right)$ has one and only one solution $\left(\mathbf{U}^{0}, \mathbf{U}^{1}, \ldots, \mathbf{U}^{M}\right)$. Thanks to the position (4.2), it suffices to show existence and uniqueness of $\mathbf{U}^{j} \in \mathbf{V}$ solving the variational equality (4.3) where $\mathbf{U}^{j-1}$ is given. In order to simplify notation, let us set $\mathbf{w}=\mathbf{U}^{j-1}$ and remove the index $j$ from $\mathbf{U}^{j}$ and $\gamma^{j}$. Actually, we will prove that there exists one and only one $N$-tuple $\left(U_{1}, \ldots, U_{N}\right)$ of functions satisfying

$$
\begin{gathered}
\left.\left(\tau_{i}+h\right) U_{i}-h \lambda_{i}^{2} \partial_{x x} U_{i}=\tau_{i} w_{i} \text { in }\right] 0, l_{i}[, \\
\partial_{x} U_{i}\left(l_{i}\right)+g_{i}\left(U_{i}\left(l_{i}\right)\right)=0 \text { for } i=1, \ldots, N, \\
U_{1}(0)=U_{2}(0)=\cdots=U_{N}(0), \\
-\sum_{i=1}^{N} a_{i} \partial_{x} U_{i}(0)=\gamma,
\end{gathered}
$$

where obviously the operators $\partial_{x}, \partial_{x x}$ now have the meaning of total derivatives. Then it will be easy to verify (arguing as in, e.g., the deduction of Eq. (2.4)) that $\mathbf{U}=\left(U_{1}, \ldots, U_{N}\right)$ is the solution of Eq. (4.3).

Our existence and uniqueness proof for the system (4.4)-(4.7) makes use of a parallel method, which consists in setting $U_{1}(0)=\cdots=U_{N}(0)=\alpha$ for an arbitrary $\alpha \in \mathbf{R}$, then solving the $N$ independent problems in (4.4)-(4.6), and finally looking for $\alpha$ such that Eq. (4.7) be satisfied. We split this argument into two parts.

LemMA 4.1. If the time step $h$ is small enough, for any $\alpha \in \mathbf{R}$ there exists one and only one $N$-tuple $\left(U_{(\alpha) 1}, \ldots, U_{(\alpha) N}\right) \in \mathbf{V}$ of functions fulfilling Eqs. (4.4)-(4.5) and such that

$$
U_{(\alpha) i}(0)=\alpha \text { for } i=1, \ldots, N .
$$

Proof. Here the index $i$ is fixed. Recalling that $\mathbf{w}=\left(w_{1}, \ldots, w_{N}\right) \in \mathbf{V}$ (cf. (4.2) and $(\mathrm{H} 1))$, let $v_{i}^{0} \in H^{3}\left(0, l_{i}\right)$ be the solution of the following linear boundary value problem:

$$
\begin{aligned}
\left(\tau_{i}+h\right) v_{i}^{0}-h \lambda_{i}^{2} \partial_{x x} v_{i}^{0} & \left.=\tau_{i} w_{i} \text { in }\right] 0, l_{i}[, \\
\partial_{x} v_{i}^{0}\left(l_{i}\right) & =0 \\
v_{i}^{0}(0) & =0 .
\end{aligned}
$$

Note that $v_{i}^{0}$ does not depend on $\alpha$. Then, by taking the difference between Eqs. (4.4) and (4.9) and accounting for the boundary conditions (4.5), (4.8), (4.10)-(4.11), 
it remains to prove that there is one and only one smooth function $v_{(\alpha) i}=U_{(\alpha) i}-v_{i}^{0}$ satisfying

$$
\begin{gathered}
\left.\left(\tau_{i}+h\right) v_{(\alpha) i}-h \lambda_{i}^{2} \partial_{x x} v_{(\alpha) i}=0 \text { in }\right] 0, l_{i}[, \\
\partial_{x} v_{(\alpha) i}\left(l_{i}\right)+g_{i}\left(v_{(\alpha) i}\left(l_{i}\right)+v_{i}^{0}\left(l_{i}\right)\right)=0, \\
v_{(\alpha) i}(0)=\alpha .
\end{gathered}
$$

Now, it is straightforward to check that any solution of Eqs. (4.12) and (4.14) can be expressed by

$$
v_{i(\alpha)}(x)=\alpha \cosh \left(m_{i} x\right)+c_{i} \sinh \left(m_{i} x\right) \quad \forall x \in\left[0, l_{i}\right],
$$

where $c_{i}$ is an arbitrary constant and

$$
m_{i}:=\sqrt{1+\tau_{i} / h} / \lambda_{i}>0 .
$$

Letting $F_{i}: \mathbf{R}^{2} \rightarrow \mathbf{R}$ be defined by $F_{i}(\beta, \xi):=m_{i}\left(\beta \sinh \left(m_{i} l_{i}\right)+\xi \cosh \left(m_{i} l_{i}\right)\right)+g_{i}\left(\beta \cosh \left(m_{i} l_{i}\right)+\xi \sinh \left(m_{i} l_{i}\right)+v_{i}^{0}\left(l_{i}\right)\right)$, it is easy to see that Eqs. (4.15) and (4.13) yield the equality

$$
F_{i}\left(\alpha, c_{i}\right)=0 .
$$

We have to prove that there exists one and only one $c_{i} \in \mathbf{R}$ solving Eq. (4.17). From (H3)-(H4) it follows that $F_{i}$ is a continuous function satisfying

$$
F_{i}(\alpha, \xi)-F_{i}(\alpha, \eta) \geq\left(m_{i} \cosh \left(m_{i} l_{i}\right)-d_{i} \sinh \left(m_{i} l_{i}\right)\right)(\xi-\eta)
$$

for any $\xi, \eta \in \mathbf{R}$ such that $\xi>\eta$. Observe also that

$$
m_{i} \cosh \left(m_{i} l_{i}\right)-d_{i} \sinh \left(m_{i} l_{i}\right)>0
$$

provided $m_{i}$ is sufficiently large, that is (cf. definition (4.16)), $h$ is sufficiently small. Letting inequality (4.18) hold, $F_{i}(\alpha, \cdot)$ is continuous, strictly increasing, and such that $F_{i}(\alpha, \eta) \rightarrow \pm \infty$ as $\eta \rightarrow \pm \infty$. Hence Eq. (4.17) has a unique solution and we can define the function

$$
c_{i}: \mathbf{R} \rightarrow \mathbf{R} \text { satisfying } F_{i}\left(\alpha, c_{i}(\alpha)\right)=0 .
$$

Then, recalling Eqs. (4.12)-(4.15), we conclude the proof of the lemma.

LEMMA 4.2. If the time step $h$ is small enough, there exists one and only one $\alpha \in \mathbf{R}$ such that

$$
-\sum_{i=1}^{N} a_{i} \partial_{x} U_{(\alpha) i}(0)=\gamma .
$$

Moreover, $\left(U_{(\alpha) 1}, \ldots, U_{(\alpha) N}\right) \in \mathbf{V}$ yields the solution of problem (4.4)-(4.7).

Proof. Letting the notation of the previous lemma hold, we set $b_{i}:=\partial_{x} v_{i}^{0}(0)$ for $i=1, \ldots, N$. Note that these values are independent of $\alpha$. Hence, owing to Eqs. (4.19) and (4.15), we can rewrite Eq. (4.20) as

$$
\sum_{i=1}^{N} a_{i} m_{i} c_{i}(\alpha)=-\gamma-\sum_{i=1}^{N} a_{i} b_{i} .
$$


We are going to prove that this equation has a unique solution for the functions $c_{i}: \mathbf{R} \rightarrow \mathbf{R}, i=1, \ldots, N$, are all continuous, strictly decreasing, and such that $c_{i}(\alpha) \rightarrow \mp \infty$ as $\alpha \rightarrow \pm \infty$. Now, the continuity of $c_{i}$ follows easily from the continuity and monotonicity properties of the function $F_{i}$ : indeed (see (4.19)) it suffices to argue as in the proof of the well-known implicit function theorem. In order to prove the other assertions, first we assume that the quantities $m_{i}$, defined by (4.16), satisfy

$$
m_{i} \tanh \left(m_{i} l_{i}\right)>d_{i} \text { for } i=1, \ldots, N .
$$

Observe that, if the time step $h$ is sufficiently small, the inequalities (4.22) hold. Also, it is not difficult to check that (4.22) implies (4.18). Then, for $\alpha>\beta$ let us show that $c_{i}(\alpha)<c_{i}(\beta)$. Assume by contradiction that $c_{i}(\alpha) \geq c_{i}(\beta)$. But, since (cf. (4.19))

$$
F_{i}\left(\alpha, c_{i}(\alpha)\right)-F_{i}\left(\beta, c_{i}(\beta)\right)=0
$$

and

$$
\alpha \cosh \left(m_{i} l_{i}\right)+c_{i}(\alpha) \sinh \left(m_{i} l_{i}\right)+v_{i}^{0}\left(l_{i}\right)>\beta \cosh \left(m_{i} l_{i}\right)+c_{i}(\beta) \sinh \left(m_{i} l_{i}\right)+v_{i}^{0}\left(l_{i}\right),
$$

with the help of (H4) and (4.18) it is straightforward to deduce that

$$
(\alpha-\beta)\left(m_{i} \sinh \left(m_{i} l_{i}\right)-d_{i} \cosh \left(m_{i} l_{i}\right)\right) \leq 0,
$$

whence, owing to (4.22), we get a contradiction. Therefore, $c_{i}$ is strictly decreasing. Now we prove a stronger inequality. Multiplying (4.23) by $(\alpha-\beta) \cosh \left(m_{i} l_{i}\right)+$ $\left(c_{i}(\alpha)-c_{i}(\beta)\right) \sinh \left(m_{i} l_{i}\right)$, from (3.3) (equivalent version of $\left.(\mathrm{H} 4)\right)$ it follows that

$$
\begin{array}{r}
m_{i}\left\{(\alpha-\beta)^{2} \sinh \left(m_{i} l_{i}\right) \cosh \left(m_{i} l_{i}\right)+(\alpha-\beta)\left(c_{i}(\alpha)-c_{i}(\beta)\right)\left(\sinh ^{2}\left(m_{i} l_{i}\right)+\cosh ^{2}\left(m_{i} l_{i}\right)\right)\right. \\
\left.+\left(c_{i}(\alpha)-c_{i}(\beta)\right)^{2} \sinh \left(m_{i} l_{i}\right) \cosh \left(m_{i} l_{i}\right)\right\} \\
\leq d_{i}\left\{(\alpha-\beta)^{2} \cosh ^{2}\left(m_{i} l_{i}\right)+2(\alpha-\beta)\left(c_{i}(\alpha)-c_{i}(\beta)\right) \sinh \left(m_{i} l_{i}\right) \cosh \left(m_{i} l_{i}\right)\right. \\
\left.+\left(c_{i}(\alpha)-c_{i}(\beta)\right)^{2} \sinh ^{2}\left(m_{i} l_{i}\right)\right\}
\end{array}
$$

By choosing $\alpha>\beta$ and eliminating some terms like

$$
\left(c_{i}(\alpha)-c_{i}(\beta)\right)^{2}\left(m_{i} \sinh \left(m_{i} l_{i}\right) \cosh \left(m_{i} l_{i}\right)-d_{i} \sinh ^{2}\left(m_{i} l_{i}\right)\right),
$$

which is positive because of (4.18), the above inequality reduces to

$$
\begin{aligned}
& m_{i}(\alpha-\beta)\left(c_{i}(\alpha)-c_{i}(\beta)\right)\left(\sinh ^{2}\left(m_{i} l_{i}\right)+\cosh ^{2}\left(m_{i} l_{i}\right)\right) \\
& \quad \leq(\alpha-\beta)^{2}\left(d_{i} \cosh ^{2}\left(m_{i} l_{i}\right)-m_{i} \sinh \left(m_{i} l_{i}\right) \cosh \left(m_{i} l_{i}\right)\right) .
\end{aligned}
$$

Hence by (4.22) there exists a positive constant $\bar{c}$, depending on $m_{i}, d_{i}, l_{i}, i=$ $1, \ldots, N$, such that

$$
c_{i}(\alpha)-c_{i}(\beta) \leq-\bar{c}(\alpha-\beta) \text { for } i=1, \ldots, N,
$$

for any $\alpha, \beta \in \mathbf{R}$ with $\alpha>\beta$. Finally, thanks to (4.24), it is easy to see that $c_{i}(\alpha) \rightarrow \mp \infty$ as $\alpha \rightarrow \pm \infty$, and the lemma is completely proved.

Thus, by means of the previous two lemmata (cf. in particular (4.22) and (4.16)), we get the following result. 
Proposition 4.1. Under the assumptions $(\mathrm{H} 1),(\mathrm{H} 3)$, and $(\mathrm{H} 4)$, there is a positive constant $\bar{h}$, depending only on $\tau_{i}, \lambda_{i}, l_{i}, d_{i}, i=1, \ldots, N$, such that for any time step $h \leq \bar{h}$ Problem $\left(\mathbf{P}_{h}\right)$ has one and only one solution.

5. A priori estimates. This section is devoted to the proof of some estimates concerning the solution of Problem $\left(\mathrm{P}_{h}\right)$. First, recalling that $h=T / M$, we introduce the approximating functions. Let $\mathbf{u}^{h} \in L^{1}(0, T ; \mathbf{V})$ and $\gamma^{h} \in L^{1}(0, T)$ be defined by

$$
\mathbf{u}^{h}(t):=\mathbf{U}^{j} \text { and } \gamma^{h}(t):=\gamma^{j} \quad \text { if }(j-1) h<t \leq j h,
$$

for $j=1, \ldots, M$. Consider also the function $\hat{\mathbf{u}}^{h} \in C^{0}([0, T] ; \mathbf{V})$ satisfying

$$
\hat{\mathbf{u}}^{h}(t):=\mathbf{U}^{j}+\frac{\mathbf{U}^{j}-\mathbf{U}^{j-1}}{h}(t-j h) \quad \forall t \in[(j-1) h, j h], j=1, \ldots, M .
$$

Owing to these positions, Eqs. (4.2), (4.3) can be rewritten (with obvious notation for the $N$ components of $\mathbf{u}^{h}$ ) as

$$
\begin{gathered}
\hat{\mathbf{u}}^{h}(0)=\mathbf{u}_{0}, \\
\left.\left\langle\partial_{t} \hat{\mathbf{u}}^{h}, \mathbf{v}\right\rangle+\left(\mathbf{u}^{h}, \mathbf{v}\right)+\sum_{i=1}^{N} a_{i} g_{i}\left(u_{i}^{h}\left(l_{i}, \cdot\right)\right) v_{i}\left(l_{i}\right)=\gamma^{h} \mathscr{T}_{0}(\mathbf{v}) \quad \forall \mathbf{v} \in \mathbf{V}, \text { a.e. in }\right] 0, T[
\end{gathered}
$$

The next statement provides estimates, independent of $h$, for the functions $\mathbf{u}^{h}$ and $\hat{\mathbf{u}}^{h}$. Recalling that $\bar{h}$ is defined by Proposition 4.1, we have the following.

Proposition 5.1. Assume that $(\mathrm{H} 1),(\mathrm{H} 2)$, and $(\mathrm{H} 4)$ hold. Then there exist two positive constants $h_{1} \leq \bar{h}$ and $C_{1}$ such that for any time step $h \leq h_{1}$ one has

$$
\begin{aligned}
& \left\|\hat{\mathbf{u}}^{h}\right\|_{H^{1}(0, T ; \mathbf{H})}^{2}+h\left\|\hat{\mathbf{u}}^{h}\right\|_{H^{1}(0, T ; \mathbf{v})}^{2} \\
& \quad+\left\|\mathbf{u}^{h}\right\|_{L^{\infty}(0, T ; \mathbf{v}) \cap L^{2}\left(0, T ; H^{2}\left(0, l_{1}\right) \times \cdots \times H^{2}\left(0, l_{N}\right)\right)}^{2} \leq C_{1} .
\end{aligned}
$$

Moreover, $h_{1}$ depends only on $\tau_{i}, \lambda_{i}, l_{i}, d_{i}, i=1, \ldots, N$.

Proof. We first deduce an auxiliary estimate which will be useful for deriving (5.5). By choosing $\mathbf{v}=\mathbf{U}^{j}$ in Eq. (4.3), it is straightforward to see that

$$
\begin{aligned}
\frac{1}{2}\left\|\mathbf{U}^{j}\right\|_{\mathbf{H}}^{2} & +\frac{1}{2}\left\|\mathbf{U}^{j}-\mathbf{U}^{j-1}\right\|_{\mathbf{H}}^{2}-\frac{1}{2}\left\|\mathbf{U}^{j-1}\right\|_{\mathbf{H}}^{2}+h\left\|\mathbf{U}^{j}\right\|_{\mathbf{V}}^{2} \\
= & -h \sum_{i=1}^{N} a_{i}\left(g_{i}\left(U_{i}^{j}\left(l_{i}\right)\right)-g_{i}(0)\right) U_{i}^{j}\left(l_{i}\right) \\
& -h \sum_{i=1}^{N} a_{i} g_{i}(0) U_{i}^{j}\left(l_{i}\right)+h \gamma^{j} \mathscr{T}_{0}\left(\mathbf{U}^{j}\right)
\end{aligned}
$$

for $j=1, \ldots, M$. With the help of (H4) (or (3.3)) and Lemma 3.1 (cf. also the proof of (3.4)) we deduce that there is a constant $C_{2}$, independent of $h$, such that

$$
-\sum_{i=1}^{N} a_{i}\left(g_{i}\left(U_{i}^{j}\left(l_{i}\right)\right)-g_{i}(0)\right) U_{i}^{j}\left(l_{i}\right) \leq \sum_{i=1}^{N} a_{i} d_{i}\left|U_{i}^{j}\left(l_{i}\right)\right|^{2} \leq C_{2}\left\|\mathbf{U}^{j}\right\|_{\mathbf{H}}^{2}+\frac{1}{4}\left\|\mathbf{U}^{j}\right\|_{\mathbf{V}}^{2},
$$


where, for instance, $C_{2}=\max \left\{d_{i} \lambda_{i}^{2} C\left(1+4 d_{i}\right) / \tau_{i}, i=1, \ldots, N\right\}$. Besides, on account of the inequality (3.1) and the definitions (2.6) and (2.3), it is easy to find a constant $C_{3}$, say $C_{3}=\left(\max \left\{\left(1+2 C \lambda_{i}^{2}\right) / a_{i}, i=1, \ldots, N\right\}\right)^{1 / 2}$, such that, for $i=1, \ldots, N$,

$$
\left|U_{i}^{j}\left(l_{i}\right)\right| \leq C_{3}\left\|\mathbf{U}^{j}\right\|_{\mathbf{v}}, \quad\left|\mathscr{T}_{0}\left(\mathbf{U}^{j}\right)\right| \leq C_{3}\left\|\mathbf{U}^{j}\right\|_{\mathbf{v}} .
$$

Then, owing to Eq. (4.1) and (H2), by applying elementary inequalities we have

$$
\begin{gathered}
\left|-\sum_{i=1}^{N} a_{i} g_{i}(0) U_{i}^{j}\left(l_{i}\right)\right| \leq\left(C_{3} \sum_{i=1}^{N} a_{i}\left|g_{i}(0)\right|\right)^{2}+\frac{1}{4}\left\|\mathbf{U}^{j}\right\|_{\mathbf{v}}^{2}, \\
\left|\gamma^{j} \mathscr{T}_{0}\left(\mathbf{U}^{j}\right)\right| \leq\left(C_{3}\|\gamma\|_{C^{0}([0, T])}\right)^{2}+\frac{1}{4}\left\|\mathbf{U}^{j}\right\|_{\mathbf{v}}^{2} .
\end{gathered}
$$

By estimating the right-hand side of Eq. (5.6) with the help of inequalities (5.7), (5.9), and (5.10), we obtain

$$
\begin{gathered}
\frac{1}{2}\left\|\mathbf{U}^{j}\right\|_{\mathbf{H}}^{2}+\frac{1}{2}\left\|\mathbf{U}^{j}-\mathbf{U}^{j-1}\right\|_{\mathbf{H}}^{2}-\frac{1}{2}\left\|\mathbf{U}^{j-1}\right\|_{\mathbf{H}}^{2}+\frac{h}{4}\left\|\mathbf{U}^{j}\right\|_{\mathbf{V}}^{2} \\
\leq 2 C_{2} h\left(\left\|\mathbf{U}^{j-1}\right\|_{\mathbf{H}}^{2}+\left\|\mathbf{U}^{j}-\mathbf{U}^{j-1}\right\|_{\mathbf{H}}^{2}\right)+h C_{4}
\end{gathered}
$$

for $j=1, \ldots, M$, where $C_{4}=\left(C_{3} \sum_{i=1}^{N} a_{i}\left|g_{i}(0)\right|\right)^{2}+\left(C_{3}\|\gamma\|_{C^{0}([0, T])}\right)^{2}$. Summing up for $j=1, \ldots, K(\leq M)$ and choosing $h_{1}=1 /\left(8 C_{2}\right)$ (in the case $C_{2}=0$, i.e., $d_{1}=\cdots=d_{N}=0$, let $\left.h_{1}=\bar{h}\right)$, by means of Eq. (4.2) and (H1) we deduce that

$$
\begin{gathered}
\frac{1}{2}\left\|\mathbf{U}^{K}\right\|_{\mathbf{H}}^{2}+\frac{1}{4} \sum_{j=1}^{K}\left\|\mathbf{U}^{j}-\mathbf{U}^{j-1}\right\|_{\mathbf{H}}^{2}+\frac{1}{4} \sum_{j=1}^{K} h\left\|\mathbf{U}^{j}\right\|_{\mathbf{V}}^{2} \\
\leq \frac{1}{2}\left\|\mathbf{u}_{0}\right\|_{\mathbf{H}}^{2}+2 C_{2} \sum_{j=0}^{K-1} h\left\|\mathbf{U}^{j}\right\|_{\mathbf{H}}^{2}+T C_{4}
\end{gathered}
$$

for any $h \leq h_{1}$ and for $K=1, \ldots, M$. Hence, by applying the discrete Gronwall lemma to the positive finite sequence $\left\{\left\|\mathbf{U}^{1}\right\|_{\mathbf{H}}^{2}, \ldots,\left\|\mathbf{U}^{M}\right\|_{\mathbf{H}}^{2}\right\}$, thanks to inequality (5.11) it is not difficult to find a constant $C_{5}$, depending only on $T, C_{2}, C_{4}$, and $\left\|\mathbf{u}_{0}\right\|_{\mathbf{H}}$, such that

$$
\left\|\mathbf{U}^{K}\right\|_{\mathbf{H}}^{2}+\sum_{j=1}^{M}\left\|\mathbf{U}^{j}-\mathbf{U}^{j-1}\right\|_{\mathbf{H}}^{2}+\sum_{j=1}^{M} h\left\|\mathbf{U}^{j}\right\|_{\mathbf{V}}^{2} \leq C_{5} \quad \text { for } K=1, \ldots, M .
$$

Now, using also inequality (5.12), we prove the stronger estimate (5.5). Taking $\mathbf{v}=\mathbf{U}^{j}-\mathbf{U}^{j-1}$ in Eq. (4.3) and summing for $j=1, \ldots, K$ with $K \leq M$, by Eq. (4.2) we infer that

$$
\begin{aligned}
& \sum_{j=1}^{K} h\left\|\frac{\mathbf{U}^{j}-\mathbf{U}^{j-1}}{h}\right\|_{\mathbf{H}}^{2}+\frac{1}{2}\left\|\mathbf{U}^{K}\right\|_{\mathbf{V}}^{2}+\frac{1}{2} \sum_{j=1}^{K}\left\|\mathbf{U}^{j}-\mathbf{U}^{j-1}\right\|_{\mathbf{V}}^{2} \\
& =\frac{1}{2}\left\|\mathbf{u}_{0}\right\|_{\mathbf{V}}^{2}+S_{g}(K)+S_{\gamma}(K),
\end{aligned}
$$


where

$$
\begin{aligned}
& S_{g}(K):=-\sum_{i=1}^{N} a_{i} \sum_{j=1}^{K} h g_{i}\left(U_{i}^{j}\left(l_{i}\right)\right) \frac{U_{i}^{j}\left(l_{i}\right)-U_{i}^{j-1}\left(l_{i}\right)}{h}, \\
& S_{\gamma}(K):=\sum_{j=1}^{K} \gamma^{j} \mathscr{T}_{0}\left(\mathbf{U}^{j}-\mathbf{U}^{j-1}\right) .
\end{aligned}
$$

We are going to estimate the terms $S_{g}$ and $S_{\gamma}$. Setting $G_{i}(\xi):=\int_{0}^{\xi} g_{i}(\eta) d \eta$ for any $\xi \in \mathbf{R}$, it is not difficult to verify that (H4) implies

$$
G_{i}(\xi) \geq g_{i}(0) \xi-d_{i} \xi^{2} / 2 \quad \forall \xi \in \mathbf{R} .
$$

Hence, on account of (5.1), (5.3), and (3.3), it is easy to see that

$$
\begin{aligned}
& S_{g}(K)=-\sum_{i=1}^{N} a_{i} \int_{0}^{K h} g_{i}\left(u_{i}^{h}\left(l_{i}, t\right)\right) \partial_{t} \hat{u}_{i}^{h}\left(l_{i}, t\right) d t \\
& =-\sum_{i=1}^{N} a_{i} \int_{0}^{K h} g_{i}\left(\hat{u}_{i}^{h}\left(l_{i}, t\right)\right) \partial_{t} \hat{u}_{i}^{h}\left(l_{i}, t\right) d t \\
& -\sum_{i=1}^{N} a_{i} \sum_{j=1}^{K} \int_{(j-1) h}^{j h}\left(g_{i}\left(u_{i}^{h}\left(l_{i}, t\right)\right)-g_{i}\left(\hat{u}_{i}^{h}\left(l_{i}, t\right)\right)\right) \frac{u_{i}^{h}\left(l_{i}, t\right)-\hat{u}_{i}^{h}\left(l_{i}, t\right)}{j h-t} d t \\
& \leq-\sum_{i=1}^{N} a_{i}\left(G_{i}\left(U_{i}^{K}\left(l_{i}\right)\right)-G^{i}\left(u_{0 i}\left(l_{i}\right)\right)\right) \\
& +\sum_{i=1}^{N} a_{i} d_{i} \sum_{j=1}^{K} \int_{(j-1) h}^{j h} \frac{\left|u_{i}^{h}\left(l_{i}, t\right)-\hat{u}_{i}^{h}\left(l_{i}, t\right)\right|^{2}}{j h-t} d t \\
& \leq \sum_{i=1}^{N} a_{i}\left(G^{i}\left(u_{0 i}\left(l_{i}\right)\right)-g_{i}(0) U_{i}^{K}\left(l_{i}\right)+\frac{d_{i}}{2}\left|U_{i}^{K}\left(l_{i}\right)\right|^{2}\right) \\
& +\sum_{j=1}^{K} \sum_{i=1}^{N} \frac{a_{i} d_{i}}{2}\left|U_{i}^{j}\left(l_{i}\right)-U_{i}^{j-1}\left(l_{i}\right)\right|^{2}
\end{aligned}
$$

for $K=1, \ldots, M$. Then, by (H1), (2.7), (5.7), (5.9), and (5.12) we infer that there is constant $C_{6}$, independent of $h$, such that

$$
S_{g}(K) \leq C_{6}+\frac{1}{4}\left\|\mathbf{U}^{K}\right\|_{\mathbf{V}}^{2}+\frac{1}{8} \sum_{j=1}^{K}\left\|\mathbf{U}^{j}-\mathbf{U}^{j-1}\right\|_{\mathbf{V}}^{2}
$$

for $K=1, \ldots, M$, where, for instance,

$$
C_{6}=\sum_{i=1}^{N} a_{i}\left|G^{i}\left(u_{0 i}\left(l_{i}\right)\right)\right|+2\left(C_{3} \sum_{i=1}^{N} a_{i}\left|g_{i}(0)\right|\right)^{2}+C_{2} C_{5} .
$$

Next, since the operator $\mathscr{T}_{0}$ is linear, it is not difficult to check that

$$
S_{\gamma}(K):=\gamma^{K} \mathscr{T}_{0}\left(\mathbf{U}^{K}\right)-\gamma^{1} \mathscr{T}_{0}\left(\mathbf{U}^{0}\right)-\sum_{j=2}^{K}\left(\gamma^{j}-\gamma^{j-1}\right) \mathscr{T}_{0}\left(\mathbf{U}^{j-1}\right),
$$


whence, owing to (5.8), (4.1), (4.2), (H1), and (H2) (cf. also (5.10)), we obtain

$$
\begin{aligned}
\left|S_{\gamma}(K)\right| \leq & \frac{1}{8}\left\|\mathbf{U}^{K}\right\|_{\mathbf{V}}^{2}+4\left(C_{3}\|\gamma\|_{C^{0}([0, T])}\right)^{2}+\frac{1}{8}\left\|\mathbf{u}_{0}\right\|_{\mathbf{V}}^{2} \\
& +C_{3} \max \left\{\left\|\mathbf{U}^{j}\right\|_{\mathbf{V}}, j=1, \ldots, M\right\} \sum_{j=2}^{K}\left|\int_{(j-1) h}^{j h} \gamma^{\prime}(t) d t\right|
\end{aligned}
$$

for $K=1, \ldots, M$, where $\gamma^{\prime} \in L^{1}(0, T)$ is the derivative of the function $\gamma$. By estimating the right-hand side of Eq. (5.13) with the help of inequalities (5.15)-(5.16), it is easy to find a constant $C_{7}$, depending only on $C_{6},\left\|\mathbf{u}_{0}\right\|_{\mathbf{V}}$, and $\|\gamma\|_{W^{1,1}(0, T)}$, such that

$$
\begin{gathered}
\sum_{j=1}^{K} h\left\|\frac{\mathbf{U}^{j}-\mathbf{U}^{j-1}}{h}-\right\|_{\mathbf{H}}^{2}+\frac{1}{8}\left\|\mathbf{U}^{K}\right\|_{\mathbf{V}}^{2}+\frac{3 h}{8} \sum_{j=1}^{K} h\left\|\frac{\mathbf{U}^{j}-\mathbf{U}^{j-1}}{h}\right\|_{\mathbf{V}}^{2} \\
\leq C_{7}+\frac{1}{32} \max \left\{\left\|\mathbf{U}^{j}\right\|_{\mathbf{V}}^{2}, j=1, \ldots, M\right\}
\end{gathered}
$$

for $K=1, \ldots, M$. Now, taking the maximum with respect to $K$ of the left-hand side of (5.17), by Eqs. (5.1), (5.2) we deduce that

$$
\begin{aligned}
& \left\|\partial_{t} \hat{\mathbf{u}}^{h}\right\|_{L^{2}(0, T ; \mathbf{H})}^{2}+\frac{3 h}{8}\left\|\partial_{t} \hat{\mathbf{u}}^{h}\right\|_{L^{2}(0, T ; \mathbf{V})}^{2}+\frac{1}{8}\left\|\mathbf{u}^{h}\right\|_{L^{\infty}(0, T ; \mathbf{v})}^{2} \\
& \quad \leq 2 C_{7}+\frac{1}{16}\left\|\mathbf{u}^{h}\right\|_{L^{\infty}(0, T ; \mathbf{V})}^{2} ;
\end{aligned}
$$

hence, recalling also inequality (5.12), we infer that

$$
\left\|\hat{\mathbf{u}}^{h}\right\|_{H^{1}(0, T ; \mathbf{H})}^{2}+h\left\|\hat{\mathbf{u}}^{h}\right\|_{H^{1}(0, T ; \mathbf{v})}^{2}+\left\|\mathbf{u}^{h}\right\|_{L^{\infty}(0, T ; \mathbf{v})}^{2} \text { is bounded }
$$

independently of $h$. Then, it is not difficult (cf. Remark 2.2) to recover from Eq. (5.4) the following equations:

$$
\tau_{i} \partial_{t} \hat{u}_{i}^{h}-\lambda_{i}^{2} \partial_{x x} u_{i}^{h}+u_{i}^{h}=0 \text { in } L^{2}\left(0, T ; H^{-1}\left(0, l_{i}\right)\right),
$$

for $i=1, \ldots, N$. Finally, owing to (5.18), by comparison inside Eq. (5.19) we have that

$$
\left\|\mathbf{u}^{h}\right\|_{L^{2}\left(0, T ; H^{2}\left(0, l_{1}\right) \times \cdots \times H^{2}\left(0, l_{N}\right)\right)} \text { is bounded independently of } h,
$$
and this concludes the proof.

REMARK 5.1. Recalling the positions (5.1), (5.2), let us point out that (5.5) implies the boundedness of $\left\|\hat{\mathbf{u}}^{h}\right\|_{C^{0}([0, T] ; \mathbf{v})}$ independently of $h$. However, we cannot deduce that the sequence $\left\{\hat{\mathbf{u}}^{h}\right\}$ is bounded in $L^{2}\left(0, T ; H^{2}\left(0, l_{1}\right) \times \cdots \times H^{2}\left(0, l_{N}\right)\right)$ for we just know that $\mathbf{u}_{0} \in \mathbf{V}$ (cf. Eq. (4.2) and (H1)).

Since we want to apply a compactnesses result in taking the limit as $h \searrow 0$, we need to consider approximating sufficiently smooth functions. Then let us conclude the present section by defining

$$
\tilde{\mathbf{u}}^{h}(t):=\hat{\mathbf{u}}^{h}(h) \quad \text { if } t \in\left[0, h\left[, \quad \tilde{\mathbf{u}}^{h}(t):=\hat{\mathbf{u}}^{h}(t) \quad \text { if } t \in[h, T]\right.\right.
$$

and observing that (see (5.5), (5.1), (5.2), and Remark 5.1)

$$
\left\|\tilde{\mathbf{u}}^{h}\right\|_{H^{1}(0, T ; \mathbf{H}) \cap C^{0}([0, T] ; \mathbf{V}) \cap L^{2}\left(0, T ; H^{2}\left(0, l_{1}\right) \times \cdots \times H^{2}\left(0, l_{N}\right)\right)} \leq C_{8}
$$

for any time step $h \leq h_{1}$, where $C_{8}$ is a constant depending only on $C_{1}$. 
6. Passage to the limit. Here, by taking the limit in Problem $\left(\mathrm{P}_{h}\right)$ as $h$ goes to 0 , we shall show that Problem (P) has at least one solution, so that (cf. Sec. 3) Theorem 2.1 will be completely proved.

In order to pass to the limit in Eqs. (5.3), (5.4), let us first remark that

$$
\gamma^{h} \rightarrow \gamma \text { strongly in } L^{2}(0, T) \text { as } h \searrow 0 .
$$

In fact, one has (cf. (5.1), (4.1), and (H2))

$$
\begin{aligned}
\left\|\gamma^{h}-\gamma\right\|_{L^{2}(0, T)}^{2} & =\sum_{j=1}^{M} \int_{(j-1) h}^{j h}|\gamma(j h)-\gamma(t)|^{2} d t \\
& =\sum_{j=1}^{M} \int_{(j-1) h}^{j h}\left|\int_{t}^{j h} \gamma^{\prime}(s) d s\right|^{2} d t \\
& \leq h \sum_{j=1}^{M}\left|\int_{(j-1) h}^{j h}\right| \gamma^{\prime}(s)|d s|^{2} \leq h\left|\sum_{j=1}^{M} \int_{(j-1) h}^{j h}\right| \gamma^{\prime}(s)|d s|^{2} \\
& =h\left\|\gamma^{\prime}\right\|_{L^{1}(0, T)}^{2}
\end{aligned}
$$

for any time step $h$. Next, by the a priori estimate (5.22) there exist $\mathbf{u}=\left(u_{1}, \ldots, u_{N}\right)$ such that, possibly taking subsequences,

$$
\begin{aligned}
& \tilde{\mathbf{u}}^{h} \rightarrow \mathbf{u} \quad \text { weakly in } H^{1}(0, T ; \mathbf{H}) \cap L^{2}\left(0, T ; H^{2}\left(0, l_{1}\right) \times \cdots \times H^{2}\left(0, l_{N}\right)\right), \\
& \tilde{\mathbf{u}}^{h} \rightarrow \mathbf{u} \quad \text { weakly star in } L^{\infty}(0, T ; \mathbf{V})
\end{aligned}
$$

as $h \searrow 0$. Since $H^{2}\left(0, l_{i}\right) \subset H^{1}\left(0, l_{i}\right) \subset L^{2}\left(0, l_{i}\right)$ with dense and compact embeddings, then, accounting for (6.3), (2.1), and (2.2), from the Aubin compactness lemma (see, e.g., $[6$, p. 58]) it follows that

$$
\tilde{u}_{i}^{h} \rightarrow u_{i} \text { strongly in } L^{2}\left(0, T ; H^{1}\left(0, l_{i}\right)\right), \quad \text { for } i=1, \ldots, N .
$$

Moreover, (6.5) and (6.4) entail

$$
\tilde{\mathbf{u}}^{h} \rightarrow \mathbf{u} \text { strongly in } L^{2}(0, T ; \mathbf{V})
$$

as $h \searrow 0$. Let us also remark that $(6.3)$ implies $u \in C^{0}([0, T] ; \mathbf{V})$ : indeed it suffices, for instance, to apply well-known interpolation results since, for $i=1, \ldots, N$, $H^{1}\left(0, l_{i}\right)$ is the intermediate space between $H^{2}\left(0, l_{i}\right)$ and $L^{2}\left(0, l_{i}\right)$.

We are going to prove that $\mathbf{u}$ is a solution of Problem (P). Since (cf. (5.1), (5.2), (5.21), and (5.5))

$$
\left\|\hat{\mathbf{u}}^{h}-\tilde{\mathbf{u}}^{h}\right\|_{L^{2}(0, T ; \mathbf{v})}^{2}+\left\|\mathbf{u}^{h}-\tilde{\mathbf{u}}^{h}\right\|_{L^{2}(0, T ; \mathbf{v})}^{2}=\frac{h}{3} \sum_{j=1}^{K}\left\|\mathbf{U}^{j}-\mathbf{U}^{j-1}\right\|_{\mathbf{v}}^{2} \leq \frac{C_{1}}{3} h,
$$

by (6.6) we easily deduce that

$$
\begin{aligned}
& \hat{\mathbf{u}}^{h} \rightarrow \mathbf{u} \quad \text { weakly in } H^{1}(0, T ; \mathbf{H}) \text { and strongly in } L^{2}(0, T ; \mathbf{V}), \\
& \mathbf{u}^{h} \rightarrow \mathbf{u} \quad \text { weakly star in } L^{\infty}(0, T ; \mathbf{V}) \text { and strongly in } L^{2}(0, T ; \mathbf{V})
\end{aligned}
$$

as $h \searrow 0$. Owing to (6.8), taking the limit in Eq. (5.3) we obtain Eq. (2.8). From 
(6.9) (cf. also (2.7)) it follow that, at least for subsequences,

$$
\left.u_{i}^{h}\left(l_{i}, \cdot\right) \rightarrow u_{i}\left(l_{i}, \cdot\right) \quad \text { strongly in } L^{2}(0, T) \text { and a.e. in }\right] 0, T[\text {. }
$$

Then, by (H3), $g_{i}\left(u_{i}^{h}\left(l_{i}, \cdot\right)\right) \rightarrow g_{i}\left(u_{i}\left(l_{i}, \cdot\right)\right)$ a.e. in $] 0, T[$. Since, on account of (6.9), we also have that $\left\|g_{i}\left(u_{i}^{h}\left(l_{i}, \cdot\right)\right)\right\|_{L^{\infty}(0, T)}$ is bounded independently of $h$, by applying the Lebesgue dominated convergence theorem we conclude that

$$
g_{i}\left(u_{i}^{h}\left(l_{i}, \cdot\right)\right) \rightarrow g_{i}\left(u_{i}\left(l_{i}, \cdot\right)\right) \text { strongly in } L^{2}(0, T)
$$

as $h \searrow 0$, for $i=1, \ldots, N$. Finally, with the help of (5.4), (6.1), (6.8), (6.9), and (6.11), it is a standard matter to check that $\mathbf{u}=\left(u_{1}, \ldots, u_{N}\right)$ solves Eq. (2.9).

REMARK 6.1. Since any weak limit point of $\left\{\tilde{\mathbf{u}}^{h}\right\}$ must coincide with the unique (cf. Sec. 3) solution u of Problem (P), the convergences (6.3)-(6.6) and (6.8), (6.9) hold for the whole sequences. Also, assuming the functions $g_{1}, \ldots, g_{N}$ are locally Lipschitz continuous in $\mathbf{R}$, it is not difficult to find a constant $C_{9}$, independent of $h$, such that

$$
\left\|\hat{\mathbf{u}}^{h}-\mathbf{u}\right\|_{C^{0}([0, T] ; \mathbf{H}) \cap L^{2}(0, T ; \mathbf{v})} \leq C_{9} h^{1 / 2} .
$$

Indeed, it suffices to take the difference between Eqs. (5.4) and (2.9), choose $\mathbf{v}=$ $\hat{\mathbf{u}}^{h}-\mathbf{u}$, and integrate in time accounting for Eqs. (5.3) and (2.8) (see Sec. 3 for an analogous procedure). Then the right-hand side can be estimated making use of (6.7), (H4), (3.1), and (6.2). However, error estimates similar to (6.12) (but accounting for the space collocation too) and details of the numerical approximation can be found in [5].

Remark 6.2. By removing the term $\left\langle\partial_{t} \mathbf{u}, \mathbf{v}\right\rangle$ from Eq. (2.9), the reduced variational equality corresponds to the steady-state problem related to $(\mathrm{P})$. Concerning this problem, by the arguments of Sec. 4 it is not difficult to see that the conditions (H3), (H4) are not sufficient to guarantee existence and/or uniqueness of a solution: indeed, it suffices to choose $g_{i}(\xi)=A_{i} \xi+B_{i}$ for arbitrary $A_{i}, B_{i} \in \mathbf{R}$ to obtain a variety of examples. Even requiring further assumptions related to the behaviour of experimental functions $g_{i}$ (see Sec. 1), it is no longer clear whether the steady-state problem has a unique solution (actually, it is easy to check that nonuniqueness may occur in the simpler voltage-clamp case).

REMARK 6.3. Given some $\mathbf{f}=\left(f_{1}, \ldots, f_{N}\right) \in L^{2}(0, T ; \mathbf{H})$, let us point out that the existence and uniqueness result stated by Theorem 2.1 still holds when the additional term $\langle\mathbf{f}, \mathbf{v}\rangle$ is included in the right-hand side of Eq. (2.9). Obviously the presence of such a term yields nonhomogeneous cable equations, that is (instead of Eq. (1.1)),

$$
\left.\tau_{i} \partial_{t} u_{i}-\lambda_{i}^{2} \partial_{x x} u_{i}+u_{i}=\tau_{i} f_{i} \text { a.e. in }\right] 0, l_{i}[\times] 0, T[.
$$

7. Regularity and final remarks. In this section we shall prove a regularity result for the solution of Problem $(\mathrm{P})$ without requiring further assumptions on the functions $g_{i}, i=1, \ldots, N$. Regarding the other data, in addition to $(\mathrm{H} 1)$ we assume that

(H5) $\exists \mathbf{v}_{0} \in \mathbf{H}:\left(\mathbf{u}_{0}, \mathbf{v}\right)+\sum_{i=1}^{N} a_{i} g_{i}\left(u_{0 i}\left(l_{i}, \cdot\right)\right) v_{i}\left(l_{i}\right)-\gamma(0) \mathscr{T}_{0}(\mathbf{v})=\left\langle\mathbf{v}_{0}, \mathbf{v}\right\rangle \quad \forall \mathbf{v} \in \mathbf{v}$,

(H6) $\gamma \in H^{1}(0, T)$. 
Observe that (see Remark 2.2) (H5) yields $u_{0 i} \in H^{2}\left(0, l_{i}\right), i=1, \ldots, N$, along with the compatibility conditions

$$
\partial_{x} u_{0 i}\left(l_{i}\right)+g_{i}\left(u_{0 i}\left(l_{i}\right)\right)=0 \quad \text { for } i=1, \ldots, N, \quad-\sum_{i=1}^{N} a_{i} \partial_{x} u_{0 i}(0)=\gamma(0) .
$$

Theorem 7.1. Assume that (H1), (H3), (H4), (H5), and (H6) hold. Then the solution $\mathbf{u}$ of Problem $(\mathbf{P})$ satisfies

$$
\mathbf{u} \in W^{1, \infty}(0, T ; \mathbf{H}) \cap H^{1}(0, T ; \mathbf{V}) .
$$

Proof. We shall show that (cf. Sec. 5) the sequence $\left\{\hat{\mathbf{u}}^{h}\right\}$ is bounded, independently of $h$, in the above space so that we shall deduce the regularity (7.1) for the weak limit u (see Sec. 6). Therefore, we are going to state another a priori estimate. We write Eq. (4.3) also for the index $(j-1)$, take the difference of the equations, and choose $\mathbf{v}=\left(\mathbf{U}^{i}-\mathbf{U}^{i-1}\right) / h$. Then on account of condition (2.3), it is a standard matter to see that

$$
\begin{aligned}
\frac{1}{2} \| & \frac{\mathbf{U}^{j}-\mathbf{U}^{j-1}}{h}\left\|_{\mathbf{H}}^{2}+h\right\| \frac{\mathbf{U}^{j}-\mathbf{U}^{j-1}}{h} \|_{\mathbf{V}}^{2} \\
\leq & \frac{1}{2}\left\|\frac{\mathbf{U}^{j-1}-\mathbf{U}^{j-2}}{h}\right\|_{\mathbf{H}}^{2}+\frac{\gamma^{j}-\gamma^{j-1}}{h} \mathscr{T}_{0}\left(\mathbf{U}^{j}-\mathbf{U}^{j-1}\right) \\
& -\frac{1}{h} \sum_{i=1}^{N} a_{i}\left(g_{i}\left(U_{i}^{j}\left(l_{i}\right)\right)-g_{i}\left(U_{i}^{j-1}\left(l_{i}\right)\right)\right)\left(U_{i}^{j}\left(l_{i}\right)-U_{i}^{j-1}\left(l_{i}\right)\right)
\end{aligned}
$$

for $j=2, \ldots, M$. On the other hand, consider Eq. (4.3) just for $j=1$ and take $\mathbf{v}=\left(\mathbf{U}^{1}-\mathbf{U}^{0}\right) / h$. With the help of (4.2) and (H5) we infer that

$$
\begin{aligned}
& \left\|\frac{\mathbf{U}^{1}-\mathbf{U}^{0}}{h}\right\|_{\mathbf{H}}^{2}+h\left\|\frac{\mathbf{U}^{1}-\mathbf{U}^{0}}{h}\right\|_{\mathbf{V}}^{2} \\
& \leq \frac{1}{2}\left\|\mathbf{v}_{0}\right\|_{\mathbf{H}}^{2}+\frac{1}{2}\left\|\frac{\mathbf{U}^{1}-\mathbf{U}^{0}}{h}\right\|_{\mathbf{H}}^{2}+\frac{\gamma^{1}-\gamma^{0}}{h} \mathscr{T}_{0}\left(\mathbf{U}^{1}-\mathbf{U}^{0}\right) \\
& \quad-\frac{1}{h} \sum_{i=1}^{N} a_{i}\left(g_{i}\left(U_{i}^{1}\left(l_{i}\right)\right)-g_{i}\left(U_{i}^{0}\left(l_{i}\right)\right)\right)\left(U_{i}^{1}\left(l_{i}\right)-U_{i}^{0}\left(l_{i}\right)\right)
\end{aligned}
$$

By summing inequalities (7.2) with respect to $j$, on account of (7.3) we have that

$$
\begin{aligned}
\frac{1}{2} \| & \frac{\mathbf{U}^{K}-\mathbf{U}^{K-1}}{h}\left\|_{\mathbf{H}}^{2}+\sum_{j=1}^{K} h\right\| \frac{\mathbf{U}^{j}-\mathbf{U}^{j-1}}{h} \|_{\mathbf{V}}^{2} \\
\leq & \frac{1}{2}\left\|\mathbf{v}_{0}\right\|_{\mathbf{H}}^{2}+\sum_{j=1}^{K} \frac{\gamma^{j}-\gamma^{j-1}}{h} \mathscr{T}_{0}\left(\mathbf{U}^{j}-\mathbf{U}^{j-1}\right) \\
& -\sum_{j=1}^{K} \frac{1}{h} \sum_{i=1}^{N} a_{i}\left(g_{i}\left(U_{i}^{j}\left(l_{i}\right)\right)-g_{i}\left(U_{i}^{j-1}\left(l_{i}\right)\right)\right)\left(U_{i}^{j}\left(l_{i}\right)-U_{i}^{j-1}\left(l_{i}\right)\right)
\end{aligned}
$$

for $K=1, \ldots, M$. Recalling inequalities (5.7), (5.8), and (5.10), it is not difficult 
to verify that

$$
\frac{\gamma^{j}-\gamma^{j-1}}{h} \mathscr{T}_{0}\left(\mathbf{U}^{j}-\mathbf{U}^{j-1}\right) \leq h\left|\frac{C_{3}}{h} \int_{(j-1) h}^{j h} \gamma^{\prime}(t) d t\right|^{2}+\frac{h}{4}\left\|\frac{\mathbf{U}^{j}-\mathbf{U}^{j-1}}{h}\right\|_{\mathbf{V}}^{2}
$$

and that

$$
\begin{gathered}
-\frac{1}{h} \sum_{i=1}^{N} a_{i}\left(g_{i}\left(U_{i}^{j}\left(l_{i}\right)\right)-g_{i}\left(U_{i}^{j-1}\left(l_{i}\right)\right)\right)\left(U_{i}^{j}\left(l_{i}\right)-U_{i}^{j-1}\left(l_{i}\right)\right) \\
\leq C_{2} h\left\|\frac{\mathbf{U}^{j}-\mathbf{U}^{j-1}}{h}\right\|_{\mathbf{H}}^{2}+\frac{h}{4}\left\|\frac{\mathbf{U}^{j}-\mathbf{U}^{j-1}}{h}\right\|_{\mathbf{V}}^{2}
\end{gathered}
$$

for $j=1, \ldots, M$. Then, by means of inequalities (7.4)-(7.6) and the Hölder inequality we easily obtain

$$
\begin{aligned}
& \left\|\frac{\mathbf{U}^{K}-\mathbf{U}^{K-1}}{h}\right\|_{\mathbf{H}}^{2}+\sum_{j=1}^{K} h\left\|\frac{\mathbf{U}^{j}-\mathbf{U}^{j-1}}{h}\right\|_{\mathbf{V}}^{2} \\
& \leq\left\|\mathbf{v}_{0}\right\|_{\mathbf{H}}^{2}+2 C_{2} \sum_{j=1}^{K} h\left\|\frac{\mathbf{U}^{j}-\mathbf{U}^{j-1}}{h}\right\|_{\mathbf{H}}^{2}+2\left(C_{3}\left\|\gamma^{\prime}\right\|_{L^{2}(0, T)}\right)^{2}
\end{aligned}
$$

for $K=1, \ldots, M$. Owing to (5.1), (5.2), (5.5), (H5), and (H6), from (7.7) it follows that

$$
\left\|\hat{\mathbf{u}}^{h}\right\|_{W^{1, \infty}(0, T ; \mathbf{H}) \cap H^{1}(0, T ; \mathbf{V})} \leq C_{10}
$$

for some constant $C_{10}$ depending only on $C_{1}, C_{2}, C_{3},\left\|\mathbf{v}_{0}\right\|_{\mathbf{H}}$, and $\left\|\gamma^{\prime}\right\|_{L^{2}(0, T)}$. Thus we conclude the proof.

REMARK 7.1. Note that (7.1) and (1.1) yield

$$
u_{i} \in L^{\infty}\left(0, T ; H^{2}\left(0, l_{i}\right)\right) \cap L^{2}\left(0, T ; H^{3}\left(0, l_{i}\right)\right)
$$

for $i=1, \ldots, N$. Indeed, it suffices to recall (2.1), (2.2) and compare the terms in (1.1). Hence, by interpolation, $\partial_{x} u_{i} \in C^{0}\left([0, T] ; H^{1}\left(0, l_{i}\right)\right) \subset C^{0}\left(\left[0, l_{i}\right] \times[0, T]\right)$, $i=1, \ldots, N$, and consequently Eq. (1.4) holds for any $t \in[0, T]$. However, let us point out that, by Eq. (1.1) and well-known statements on parabolic equations, the solution $\mathbf{u}$ of Problem (P) satisfies $u_{i} \in C^{\infty}(] 0, l_{i}[\times] 0, T[)$ independently of the regularity of $\mathbf{u}_{0}, \gamma, g_{i}, i=1, \ldots, N$. Then, under the assumptions of Theorem $7.1,\left(u_{1}, \ldots, u_{N}\right)$ is a classical solution of system (1.1)-(1.5).

REMARK 7.2. If the term $\langle\mathbf{f}, \mathbf{v}\rangle$ is added in the right-hand side of Eq. (2.9) (cf. Remark 6.3), (7.1) still holds provided that $\mathbf{f} \in W^{1,1}(0, T ; \mathbf{H})$. Obviously, in this case it is no longer true (see Eq. (6.13)) that $u_{i} \in C^{\infty}(] 0, l_{i}[\times] 0, T[)$ for $i=1, \ldots, N$.

REMARK 7.3. The results contained in this paper extend to the case of functions $g_{1}, \ldots, g_{N}$ depending also on the time. Concerning this setting, it is not difficult to check that a sufficient condition (replacing $(\mathrm{H} 3),(\mathrm{H} 4)$ ) is given, for instance, by the following:

$$
g_{i} \in C^{0}(\mathbf{R} \times[0, T]) \quad \text { satisfies } \quad g_{i}(\xi, t)-g_{i}(\eta, t) \geq-d_{i}(\xi-\eta)
$$

for any $\xi>\eta$ and $t \in[0, T], i=1, \ldots, N$. 


\section{REFERENCES}

[1] P. Asher and L. Nowak, The role of divalent cations in the $N$-methyl-D-aspartate responses of mouse central neurones in culture, J. Physiol. 399, 247-266 (1988)

[2] R. FitzHugh, Dimensional analysis of nerve models, J. Theoret. Biol. 40, 517-541 (1973)

[3] V. Comincioli, E. D'Angelo, D. Funaro, P. Rossi, and A. Torelli, A mathematical model of potential spreading along neuron dendrites of cerebellar granule cells, Appl. Math. Comput. 59, 73-87 (1993)

[4] E. D'Angelo, P. Rossi, and J. Garthwaite, Dual-component NMDA receptor currents at a single central synapse, Nature 346, 467-469 (1990)

[5] D. Funaro, Approximation by the Legendre collocation method of a model problem in electrophysiology, J. Comput. Appl. Math. 43, 261-271 (1992)

[6] J. L. Lions, Quelques méthodes de résolution des problèmes aux limites non linéaires, Dunod Gauthier-Villars, Paris, 1969

[7] M. L. Mayer, G. L. Westbrook, and P. B. Guthrie, Voltage dependent block by $\mathrm{Mg}^{2+}$ of NMDA responses in spinal cord neurons, Nature 309, 261-263 (1984)

[8] L. Nowak, P. Bregestowsky, P. Asher, A. Herbet, and A. Prochiantz, Magnesium gates glutamate-activated channels in mouse central neurones, Nature 307, 462-465 (1984)

[9] S. Palay and V. Chan-Palay, Cerebellar cortex, Springer-Verlag, Berlin, 1974

[10] M. Palkovits, P. Magyar, and J. Szentagothai, Quantitative hystological analysis of the cerebellar cortex in the cat. IV. Mossy fiber-Purkinje cell numerical transfer, J. Brain Res. 45, 15-29 (1972)

[11] W. Rall, Core conductor theory and cable properties of neurons, Handbook of Physiology. The Nervous System. Cellular Biology of Neurons, Amer. Physiol. Soc., Bethesda, MD, 1981, pp. 39-97 